\title{
Four hours of veno-venous extracorporeal membrane oxygenation using bi-caval cannulation affects kidney function and induces moderate lung damage in a mouse model
}

\author{
Ruslan Natanov ${ }^{1 \dagger}$, Abdurasul Khalikov ${ }^{1 \dagger}$, Faikah Gueler ${ }^{2}$, Ulrich Maus ${ }^{3}$, Erin C. Boyle ${ }^{1}$, Axel Haverich ${ }^{1}$, \\ Christian Kühn ${ }^{1}$ and Nodir Madrahimov ${ }^{1,4^{*}}$ (D)
}

\author{
* Correspondence: Madrahimov_N@ \\ ukw.de \\ ${ }^{\dagger}$ Ruslan Natanov and Abdurasul \\ Khalikov contributed equally to this \\ work. \\ 'Department of Cardiothoracic, \\ Transplantation and Vascular \\ Surgery, Hannover Medical School, \\ Hannover, Germany \\ ${ }^{4}$ Clinic of Cardiothoracic Surgery, \\ University Clinic of Würzburg \\ Oberdürrbacher Strasse 6, 97080 \\ Würzburg, Germany \\ Full list of author information is \\ available at the end of the article
}

\begin{abstract}
Background: Improvement of single site cannulation for extracorporeal membrane oxygenation (ECMO) therapy is pivotal for reduction of patient morbidity and mortality in respiratory failure. To further improve the cardiopulmonary outcomes and reduce end organ damage, we established a murine model for single site cannulation with a double lumen cannula.

Results: We created a hemodynamically stable double lumen cannula and successfully implanted it through the jugular vein into the upper and lower vena cava. This allowed adequate drainage of the blood. Blood gas analysis showed excellent oxygenation and $\mathrm{CO}_{2}$ reduction. There was no excessive bleeding. No signs of right heart congestion were present which was confirmed in the histological analysis of the liver. Histology demonstrated moderate lung damage and mild acute kidney injury. Neutrophil infiltration was similar in ECMO and sham kidneys.

Conclusions: Veno-venous extracorporeal circulation deteriorates kidney function and promotes moderate pulmonary damage.
\end{abstract}

Keywords: Mouse model, Extracorporeal membrane oxygenation, Single cannula, Double lumen cannula

\section{Introduction}

Extracorporeal membrane oxygenation (ECMO) is an essential tool in cardiorespiratory failure [1]. In isolated respiratory failure, veno-venous (vv)-ECMO is the therapy of choice as bridge to either recovery or transplantation [2]. In this setting, there are two cannulas placed in the veins of the patient. The draining cannula is typically placed through the femoral vein and advanced via the inferior vena cava to the right atrium. The returning cannula is typically placed in the right jugular vein and advanced to the border of the right atrium and upper vena cava. Through the membrane oxygenator, blood is supplied with oxygen and carbon dioxide is washed out. Indications for vv-ECMO implementation include severe acute respiratory distress syndrome

(c) The Author(s). 2019 Open Access This article is distributed under the terms of the Creative Commons Attribution 4.0 International License (http://creativecommons.org/licenses/by/4.0/), which permits unrestricted use, distribution, and reproduction in any medium, provided you give appropriate credit to the original author(s) and the source, provide a link to the Creative Commons license, and indicate if changes were made. 
(ARDS), exacerbation of chronic obstructive pulmonary disease (COPD), and as a bridge to lung transplantation [3, 4]. Although this system is highly effective, complications including bleeding, pneumothorax, and cannula infections have been reported during cannulation and ongoing ECMO therapy $[2,4,5]$. Moreover, patients on awake ECMO are difficult to mobilize when the femoral vein is cannulated. The use of a double lumen cannula has been proposed to optimize physiotherapy, patient mobilization, and patient comfort [6-8]. Additionally, placement of a double lumen cannula at a single site is less traumatic and has been proven to be very effective when $\mathrm{CO}_{2}$ elimination is needed. Major limitations of this system include reduced venous backflow [9], relatively low blood flow [10], cannula displacement, and thrombosis [11].

To further improve patient outcomes and reduce morbidity, a reliable and reproducible animal model is needed for research purposes. Based on our previously established mouse model of vv-ECMO [12], here we describe a novel murine double lumen cannula for vv-ECMO support.

\section{Material and methods}

Animals

Nineteen male C57Bl/6 mice were obtained from Charles River (Sulzfeld, Germany) and used for the experiments. Animals were randomly divided into sham-operated $(n=6)$ animals, $4 \mathrm{~h} \mathrm{vv-ECMO}(n=8)$ animals, and animals used in the design of the double lumen cannula ( $n=5$, see below). The weight of mice ranged between 25 and $35 \mathrm{~g}$. This study was performed in compliance with the German Animal Protection Law (TierSchG) and was approved by the local animal welfare committee (Lower Saxony State Office for Consumer Protection and Food Safety, Protocol TSA 33.12-42502-04-16/2250).

\section{Design and construction of the double lumen cannula}

A 2F double lumen silicone-based catheter (Vygon GmbH \& CO.KG Medizintechnik, Aachen, Germany) was used as the basis for the production of the double lumen cannula (Fig. 1). Using a sharp blade, outflow fenestrations were made in the catheter at the eventual site of the superior vena cava and inferior vena cava (Fig. 2). Similarly, inflow fenestrations were made at the height of the right atrium. To ensure optimal positioning of the fenestrations, multiple measurements were performed on five mouse cadavers of a similar age/size to the experimental mice. For optimal drainage and minimal shunting, venous outflow fenestrations were made $0.2 \mathrm{~mm}, 0.4 \mathrm{~mm}$, and $20 \mathrm{~mm}$ from the distal end. Atrial inflow fenestrations were made $0.4 \mathrm{~mm}$ and $0.5 \mathrm{~mm}$ from the distal end. To prevent recirculation and shunting of the blood, the distal end of the inflow cannula lumen was sealed.

\section{Surgical procedure and extracorporeal membrane oxygenation}

Surgical preparation (Fig. 3) and ECMO setup were carried out as previously described [12]. In brief, all animals were anesthetized with isoflurane mask narcosis (Fig. 3 (a)) and spontaneous breathing was maintained. Subcutaneous carprofen (Zoetis, Parsippany, NJ, USA) injections were given as additional analgesia ( $5 \mathrm{mg} / \mathrm{kg}$ body weight). Perfusion solution consisted of a 1:1 solution of Tetraspan: Sterofundin (B Braun Medical, Melsungen, Hesse, Germany) that had been heparinized (30 IU $/ \mathrm{ml})$. Prior to 


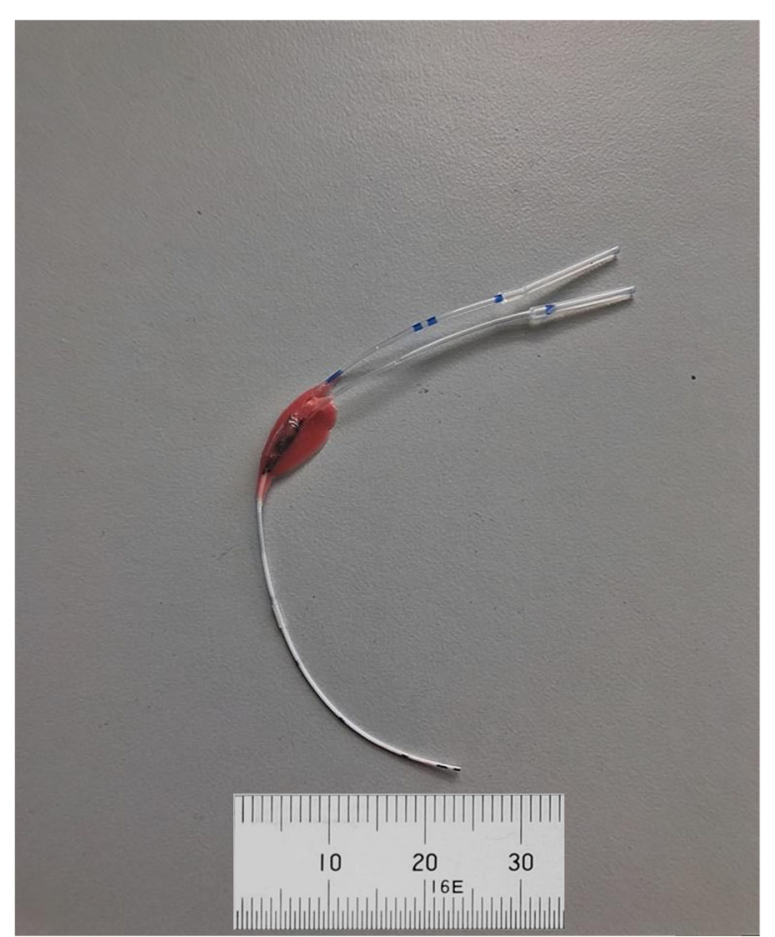

Fig. 1 The two French double lumen cannula which were the basis for the double lumen single ECMO cannula. Ruler for reference in $\mathrm{mm}$

cannulation, the ECMO circuit was primed with $500 \mu \mathrm{l}$ of perfusion solution. Buffering of the solution was carried out using $2.5 \% \mathrm{v} / \mathrm{v}$ of an $8.4 \%$ solution of sodium bicarbonate. An arterial pressure line in the left femoral artery was used for blood sampling and blood pressure monitoring.

For cannulation, a lateral skin incision on the left side of the neck was made to expose the left jugular vein. An 8-0 silk suture was placed cranially to ligate the distal segment, and a slip knot was placed at the proximal end of the vein. After introducing the double lumen cannula into the left jugular vein (Fig. 3 (b)), it was moved $3.5 \mathrm{~cm}$ in the direction of the superior vena cava, and further into the inferior vena cava. The cannula was then secured using slip-knots. After confirmation of correct position of the cannula, extracorporeal circulation was started and continued for $4 \mathrm{~h}$. In the sham group, the surgical procedure for cannulation was identical to the ECMO animals; however, no extracorporeal circulation was commenced. In both ECMO- and sham-treated animals, the procedure was performed for $4 \mathrm{~h}$.

\section{Blood gas analysis}

Blood gas analysis (BGA) was performed from blood sampled from the femoral artery to evaluate the oxygenation and metabolic state of animals undergoing $\mathrm{vv}$-ECMO.

\section{Blood tests of kidney and liver function}

Blood samples were collected via the femoral artery prior to the procedure and via exsanguination after termination of the experiment. Serum was stored at $-20{ }^{\circ} \mathrm{C}$ for later 


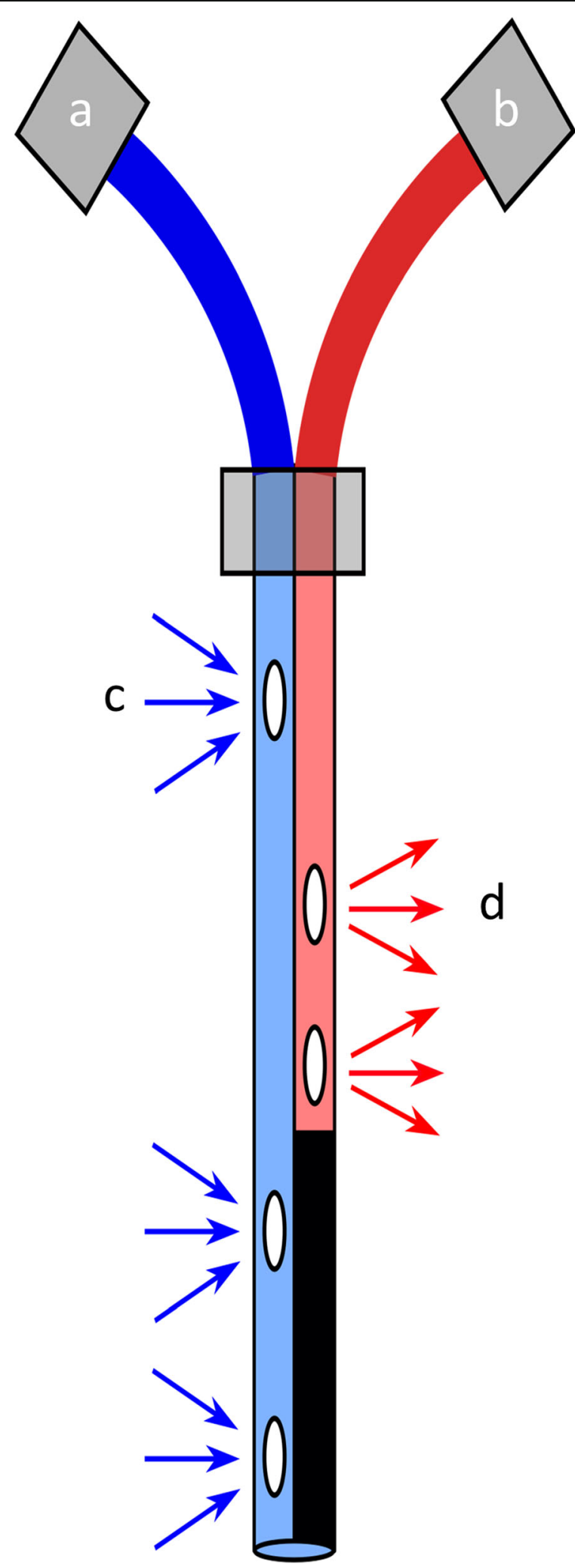

Fig. 2 Schematic depiction of the double lumen silicone cannula. Two lines were constructed into a single cannula. One line extracts blood (a) from the upper and lower vena cava before passing it through the oxygenator and giving the blood back in the right atrium (b). Blue (c) and red arrows (d) show the fenestrations in the cannula 


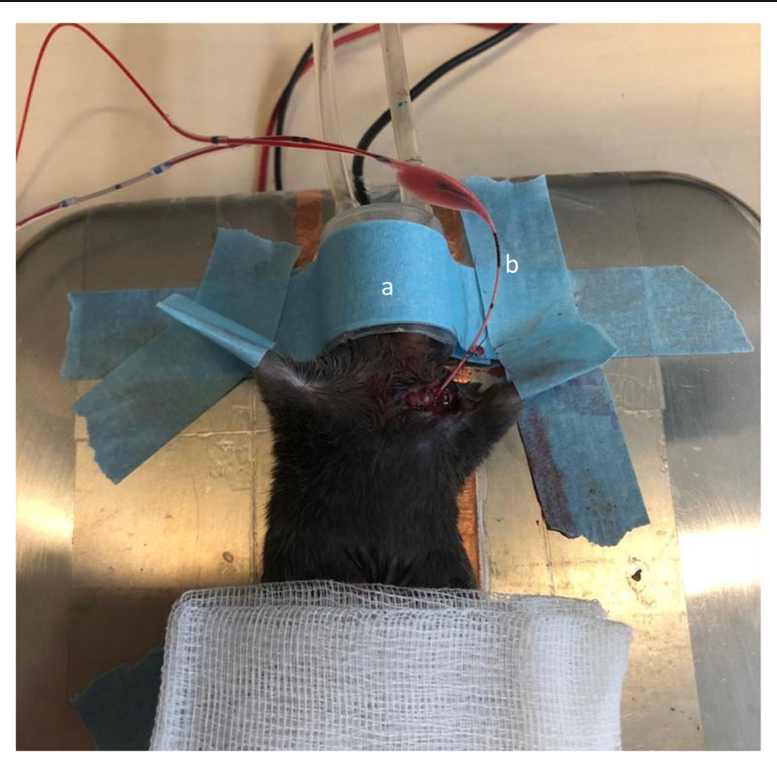

Fig. 3 Experimental setup with mask narcosis (a) and double lumen cannulation through the jugular vein (b). During vv-ECMO, blood was circulated via a self-made oxygenator and returned via the right atrium

analysis. Clinical chemistry was done using an Olympus analyzer (AU 400) according to the manufacturer's instructions to evaluate liver enzymes (glutamate oxaloacetate transaminase (GOT) and glutamate pyruvate transaminase (GPT)) and kidney function (creatinine, urea) after vv-ECMO. All values were corrected for hemodilution as previously described [13]. Furthermore, to evaluate hemolysis during the experiment, lactate dehydrogenase $(\mathrm{LDH})$ values were compared between the start of the experiment and after 4 hours.

\section{Histology}

For organ fixation, lungs were filled with $4 \%$ paraformaldehyde via injection into the trachea. Filled lungs were explanted and incubated in $4 \%$ formalin overnight at $4{ }^{\circ} \mathrm{C}$. After dehydration and deparaffinization, lungs were stained with hematoxylin and eosin (H\&E) and histologically assessed for pulmonary damage. The liver and kidney were also collected, fixed in $4 \%$ paraformaldehyde, and stored for $24 \mathrm{~h}$ at $4{ }^{\circ} \mathrm{C}$. Twomicrometer paraffin sections were stained with periodic acid-Schiff (PAS) to evaluate kidney and liver morphology. Assessment of acute kidney injury (AKI) and liver damage was done using a method previously described [13].

\section{Statistics}

Statistical analyses were performed using GraphPad Prism version 5.0 software (GraphPad Software Inc., San Diego, CA, USA). The Kolmogorov-Smirnov test revealed the data was normally distributed. One-way ANOVA with post hoc Bonferroni tests were used for statistical analysis. Unless otherwise stated, data are presented as mean \pm standard deviation (SD). 


\section{Results}

\section{Hemodynamics and oxygenation after 4-h sham and ECMO treatment}

During the experiments, stable hemodynamics were observed. No excessive blood loss was noted, and this was confirmed by the relatively stable hematocrit (Hct) and hemoglobin $(\mathrm{Hb})$ values. Sham-operated animals had an initial Hct value of $42.5 \pm$ $0.9 \%$, which dropped after $4 \mathrm{~h}$ to $38.3 \pm 0.9 \%$ (not significant, n.s.) due to regular blood samplings (Fig. 4a). In general, approximately $150 \mu$ l of blood was taken over the course of the experiment to analyze BGA in both arterial and venous blood. There was a similar drop in Hct seen in the vv-ECMO-treated group with an initial value of $24.5 \pm 1.3 \%$ and an end value of $18.3 \pm 1.7 \%(p=0.027)$ (Fig. 4a). A significantly lower hematocrit was measured after $4 \mathrm{~h}$ of ECMO in comparison to $4 \mathrm{~h}$ sham $(p=0.0016)$ (Fig. $4 \mathrm{a})$. These findings were comparable to our previous data [14], and the lower values were due to hemodilution.

Hemoglobin showed a similar drop over the course of the experiments. The initial level of $\mathrm{Hb}$ of $13.6 \pm 0.2 \mathrm{~g} / \mathrm{dL}$ in the sham group was in the normal range and significantly dropped during the experiment to $12.2 \pm 0.3 \mathrm{~g} / \mathrm{dL}(p=0.003)$ due to blood sampling (Fig. 4b). Although not statistically significant, a fall in hemoglobin from $8.7 \pm 0.4$ to $7.2 \pm 0.4 \mathrm{~g} / \mathrm{dL}$ was observed in the ECMO treatment group $(p=0.059)$, probably due to hemolysis caused by the ECMO.

To ensure proper membrane oxygenator function, blood sampling was performed directly before and after the oxygenator following initiation of vv-ECMO. Preoxygenator $\mathrm{pO}_{2}(113.0 \pm 14.1 \mathrm{mmHg})$ and post-oxygenator $\mathrm{pO}_{2}(680.9 \pm 19.2$ $\mathrm{mmHg})$ showed a significant increase $(p<0.0001)$ indicating an excellent oxygenation capacity of our membrane oxygenator (Fig. 5a). To evaluate animal oxygenation, arterial BGAs were taken at the beginning of the experiment and were compared to BGAs taken after $4 \mathrm{~h}$. Once initiated, ECMO was associated with high pO2 levels, which did not significantly change during the 4-h course of ECMO ( $p$ $=0.09$ ) (Fig. 5b). After $4 \mathrm{~h}$, the ECMO group showed a significantly higher arterial $\mathrm{pO}_{2}(567.1 \pm 56.1 \mathrm{mmHg})(p<0.001)$ compared to the sham group $(312.0 \pm 23.4$ $\mathrm{mmHg}$ ) (Fig. 5b). No significant differences in arterial pCO2 were recorded
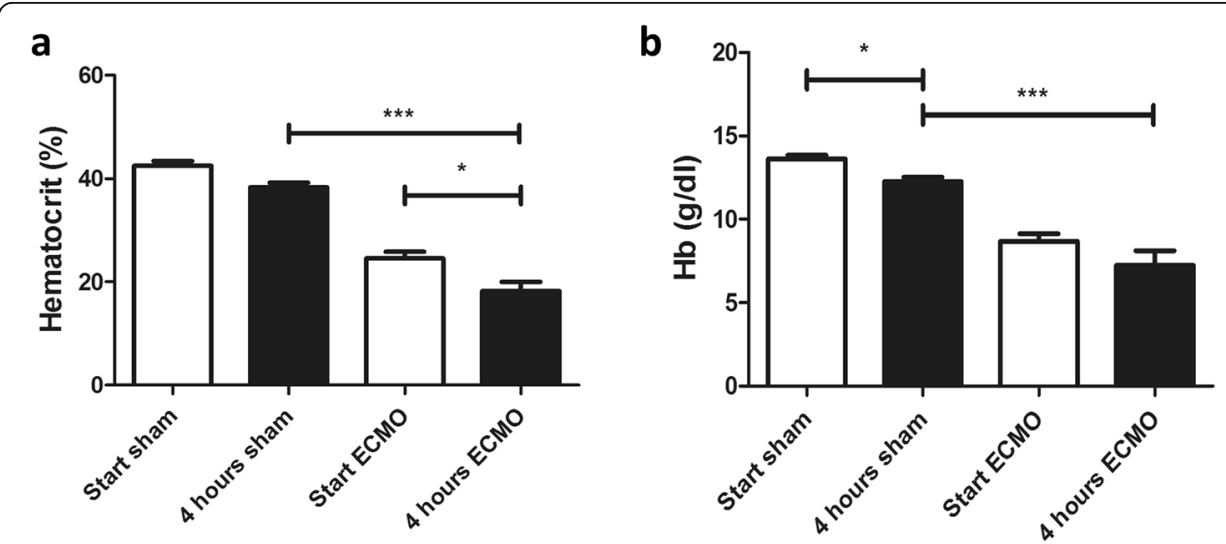

Fig. 4 Hematocrit (a) and hemoglobin (b) values of sham-operated $(n=6)$ and 4-hour ECMO-treated $(n=$ 8) animals significantly decreased over the course of the experiment. All values are given as mean \pm SD.* $p$ $<0.05$; ${ }^{* *} p<0.01$, ${ }^{* *} p<0.0001$, ns not significant 
between the start and end of the experiment in the sham or ECMO groups (Fig. $5 b)$. Similarly, no significant differences in venous $\mathrm{pH}$ or $\mathrm{sO}_{2}$ were observed between the start and end of the experiment for sham- or ECMO-treated animals (data not shown).

Venous blood taken from the inferior vena cava showed better $\mathrm{pO}_{2}$ values after 4-h ECMO therapy $(p=0.036)$ compared to the sham animals (Fig. $5 \mathrm{c}$ ). There was no significant rise in venous blood $\mathrm{pO}_{2}$ after 4-h ECMO compared to start of the experiment $(p=0.17)$. No significant decrease in $\mathrm{pCO}_{2}$ values was seen over the course of $4 \mathrm{~h}$ in either sham- or ECMO-treated animals (Fig. 5d, e).

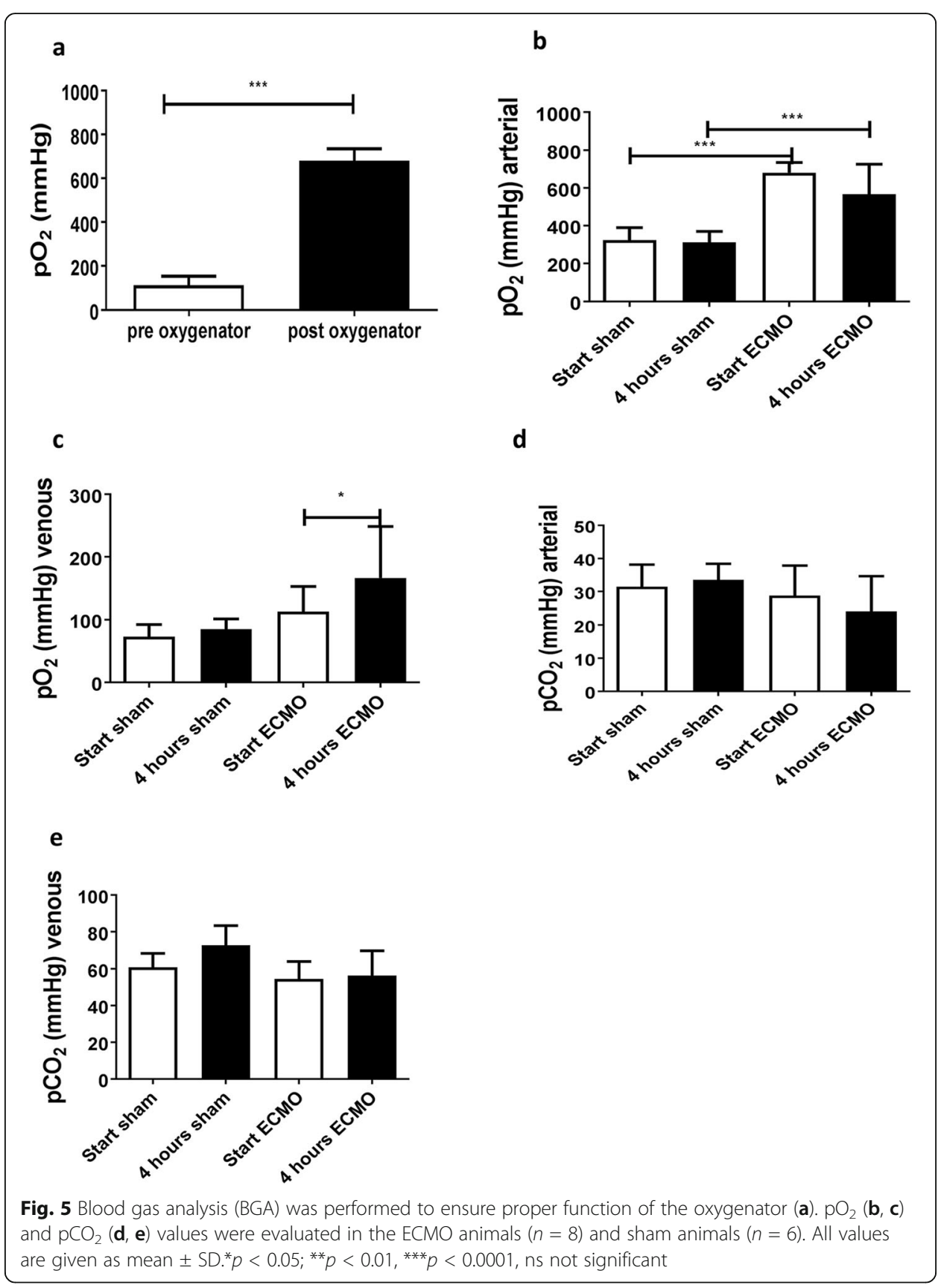




\section{Evaluation of organ damage after 4-h sham or ECMO treatment}

Four hours after either sham operation or vv-ECMO with the double lumen single cannulation technique, organ damage was evaluated. No pulmonary pathology was seen after $4 \mathrm{~h}$ in the sham group (Fig. 6a, c, e). Although the ECMO-treated animals showed regular bronchial and vascular architecture, small peripheral vessel coagulopathy was more frequently observed compared to sham-operated animals (Fig. 6).

Next, renal function and kidney histology were assessed. Significant differences in serum creatinine and urea $(p<0.001)$ were seen after ECMO treatment compared to sham-operated animals (Fig. 7a, b). These differences may be due to either pre-renal acute kidney injury or hemolysis. Quantitative scoring (Fig. 7c) and histological evaluation (Fig. 7d, e) of the kidney showed a significant increase in the AKI $(p=0.040)$ score in the ECMO group, suggesting decreased renal function after vv-ECMO.

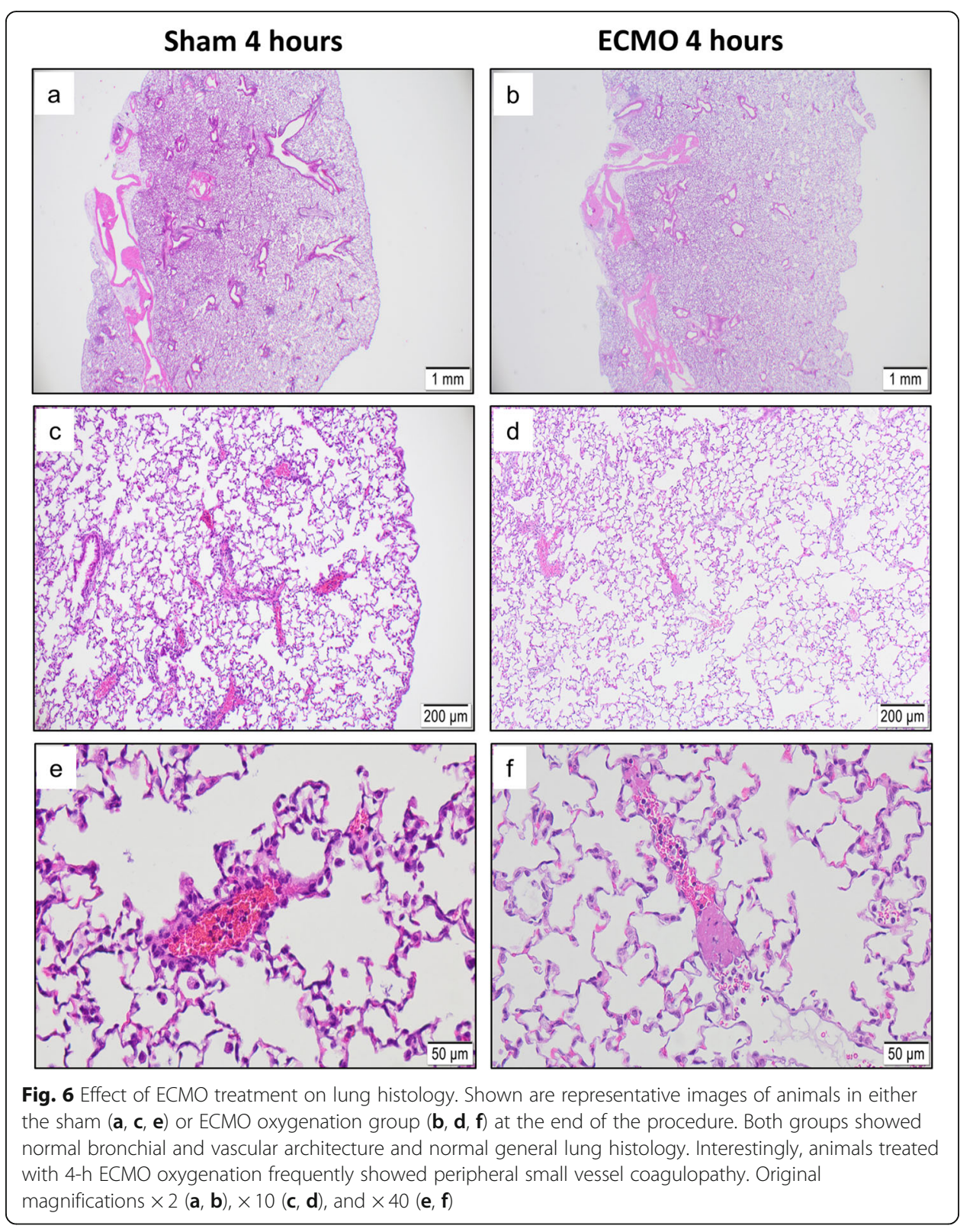




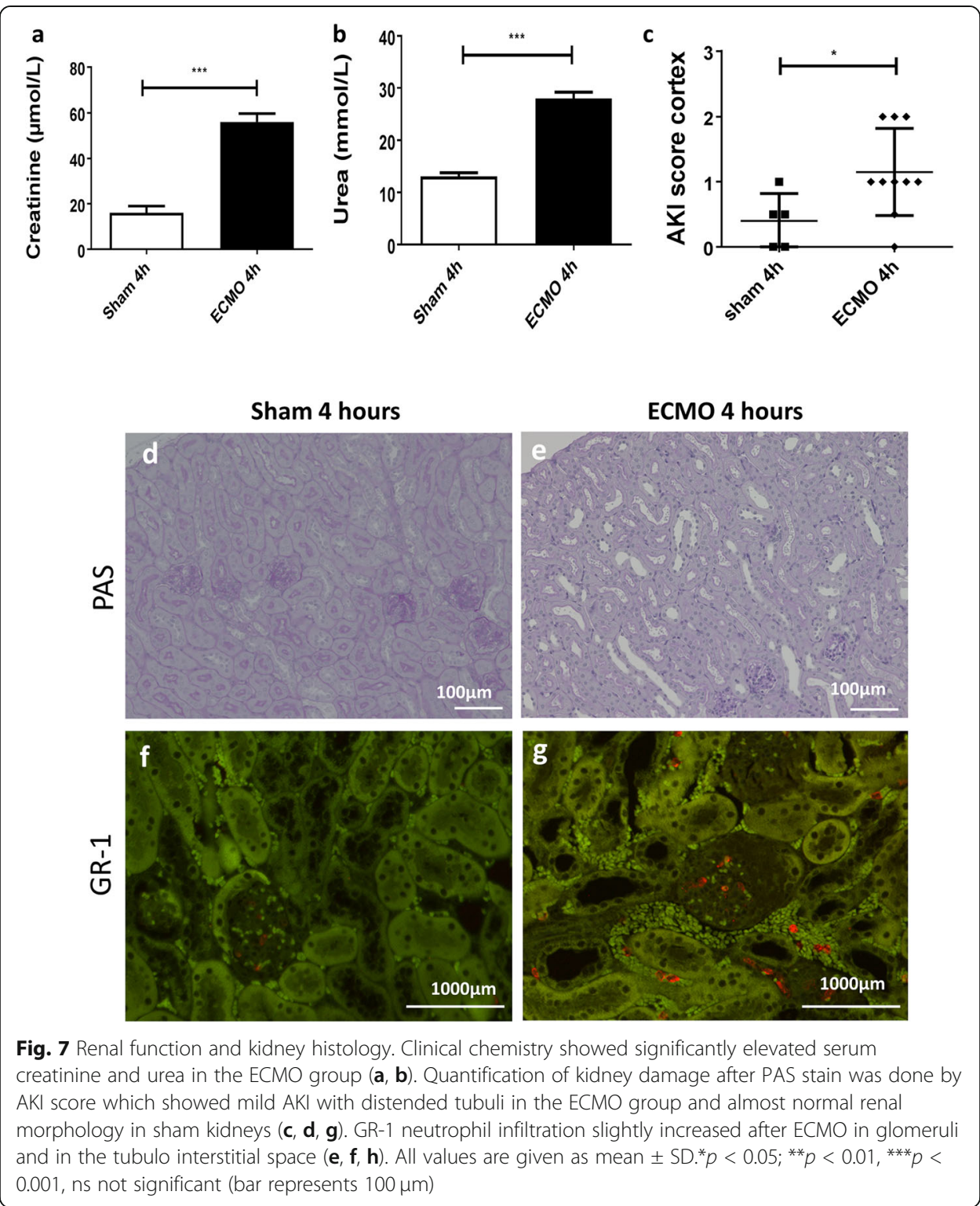

Furthermore, although more GR-1 staining due to neutrophil infiltration was seen in the kidney after 4-h ECMO compared to sham-treated animals (Fig. 7f, g), quantitative scoring did not reach significance $(p=0.845)$.

After $4 \mathrm{~h}$, blood tests for liver function revealed no significant differences between ECMO- and sham-treated animals (Fig. 8a, b). Congestive hepatopathy can occur upon congestive heart failure, but this data suggests right heart congestion did not occur during the procedure. No histological abnormalities were seen in the H\&E- and PASstained cardiac samples (data not shown). As another measure of tissue damage, LDH was measured in the blood. After $4 \mathrm{~h}$, a significant increase in LDH concentration was seen in ECMO-treated animals compared to sham treatment $(p<0.001)$ (Fig. 8c).

Liver pathology was evaluated using PAS staining and showed various extents of irregular glycogen loss in all animals (Fig. 8d, e). However, there were no signs of necrosis, cellular infiltration, or edema in liver tissue. Furthermore, quantification of 

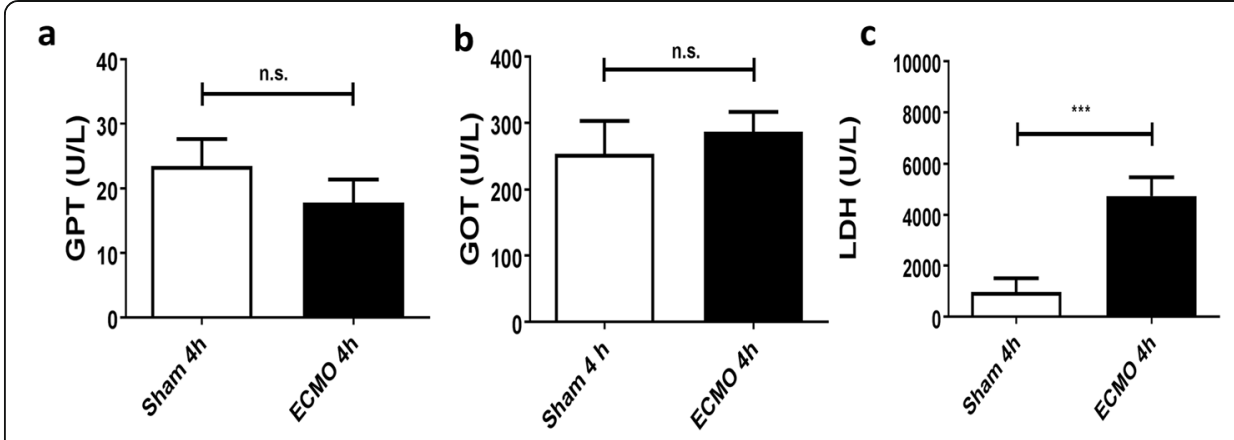

Sham 4 hours

ECMO 4 hours
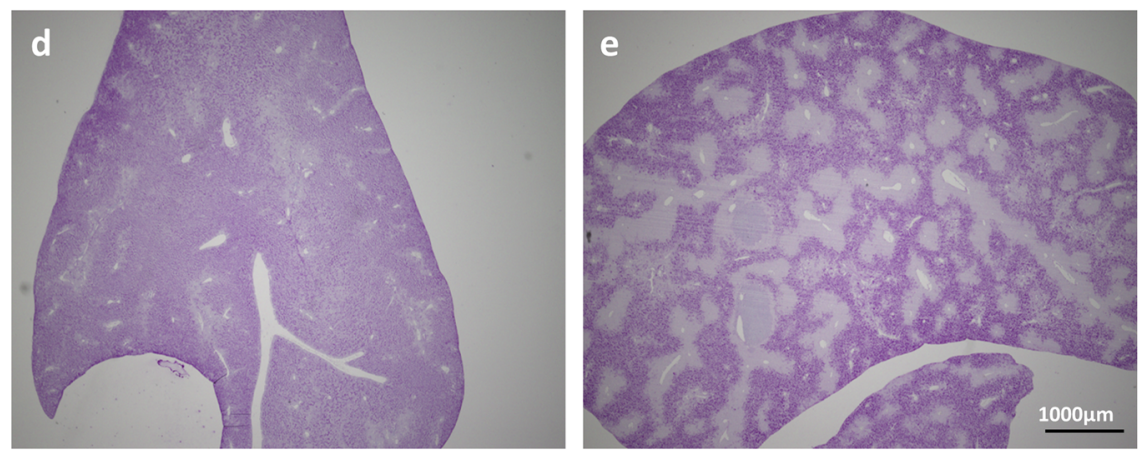

f

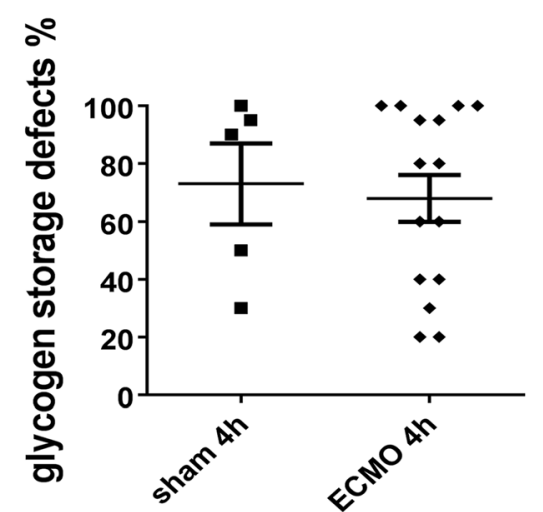

Fig. 8 Clinical chemistry and histology of the liver. Blood samples taken after $4 \mathrm{~h}$ showed no significant elevation in GOT and GPT in the ECMO animals when compared to sham animals (a, b). LDH was significantly elevated in the ECMO treatment group compared to the sham group (c). Although histological evaluation of the liver did show reduced glycogen storage capacity in the ECMO group (d, e) compared to the sham group, this was not statistically significant (f). All values are given as mean \pm SD. ${ }^{*} p<0.05$; ${ }^{* *} p<$ $0.01,{ }^{* * *} p<0.0001$, ns not significant (bar represents $1000 \mu \mathrm{m}$ )

glycogen storage defects (Fig. 8f) did not show a significant difference in either treatment group $(p=0.75)$.

\section{Discussion}

Animal models of bi-caval ECMO are rare, with to date, only two swine models being described $[15,16]$. To our knowledge, no papers on bi-caval double lumen ECMO in a murine model have been published, making this a novel model. Although the classic two cannula vv-ECMO has become increasingly popular in patients with respiratory 
failure, this modality has its limitations. In small pediatric patients, there is no possibility for a second cannula as the femoral veins are often too small for cannulation. As previously stated, patient immobilization due to ECMO makes physical therapy practically impossible and may prolong the intensive care unit stay. In awake ECMO patients, ECMO tolerability is much greater and was not associated with an increase in complications such as bleeding $[8,10]$. For this group of patients, a bi-caval double lumen cannula has been proposed as a therapeutic option [17]. This single cannula is inserted via the jugular vein and is ideally positioned using transesophageal echocardiography [18]. The main objective of vv-ECMO is carbon dioxide $\left(\mathrm{CO}_{2}\right)$ elimination and, in part, supportive oxygenation in respiratory failure. Depending on the blood flow and diameter of the cannula, the rate of $\mathrm{CO}_{2}$ elimination can be regulated. Our new small animal model of double lumen ECMO is a proof of concept of the technique for potential future use to explore the pathomechanisms of SIRS, respiratory compensation in acute or end-stage lung disorders, and other systemic complications purely related to the blood damage caused by extracorporeal circulation. Additionally, there are commercially available genetically modified mice that develop, for example, respiratory insufficiency directly after birth or lung fibrosis in older age, in which ECMO could be studied in the context of these underlying diseases. Moreover, our cannulation technique can be successfully used in other experimental models involving dialysis, cytokine adsorbtion, plasmapheresis, or hemadsorbtion due to a similarity in function with other venous double lumen catheters widely used in the clinic.

Our results showed a feasible, hemodynamically stable setup with proper oxygenation and a trend of decreasing $\mathrm{CO}_{2}$ values. This suggests an optimized functioning of the oxygenator and a minimal shunting of the ECMO blood. No clinical signs of heart congestion were seen. Furthermore, clinical chemistry showed no increase in GOT and GPT values, thus making right heart congestion less probable. Increases in creatinine and urea values suggest a decrease in kidney function. Simultaneously, a significant increase in LDH was seen which may be explained due to hemolysis during ECMO. Hemolysis has previously been associated with impaired acute kidney failure after cardiopulmonary bypass [19]. Kidney failure during extracorporeal circulation is common and has multiple causes. In our model, the AKI was apparent with tubular damage upon histological assessment. Previous work showed an association between tubular damage and hemolysis, reduced renal blood flow, and hypoxia in ECMO [20-24]. AKI is an expected clinically relevant consequence of ECMO, and therefore, our model can be used to explore therapeutic interventions to prevent organ damage. In addition, due to the availability of a large number of genetically modified mouse strains, the model could also be used to investigate the molecular mechanisms leading to ECMO-related organ damage. As it is often difficult to distinguish whether complications seen clinically are caused by ECMO itself or arise from the underlying disorders accompanying acute and end-stage diseases, our model will allow researchers to specifically study the effects of ECMO on a healthy organism.

Our model did not show a significant drop in $\mathrm{CO}_{2}$ concentration in the arterial BGA over the course of a 4-h ECMO procedure. We believe this was due to spontaneous breathing of the animals. However, a clear trend in $\mathrm{CO}_{2}$ reduction was observed. A further reduction in $\mathrm{CO}_{2}$ values may be achieved by increasing gas flow or EMCO blood flow. Inducing a hypercapnic state in a mouse model would 
be beneficial to address this issue. As there are no small animal models available, we could not compare these findings. Large animal ECMO models, however, showed similar oxygenation results and comparable hemodynamics but did not report renal or hepatic function parameters $[15,16]$. In conclusion, we present the proof-of-concept use of bi-caval double lumen ECMO in a murine model. Future projects include application of this model in a murine lung disease model to determine the outcome using different ECMO modalities.

\section{Conclusion}

Veno-venous extracorporeal membrane oxygenation elicits a decrease in renal function and significantly more histological renal damage. Furthermore, it induces pulmonary morphological changes after $4 \mathrm{~h}$ in a murine model. Liver function and histology does not seem to be affected, neither were there signs of right heart congestion.

\section{Abbreviations}

AKI: Acute kidney injury; ARDS: Acute respiratory distress syndrome; BGA: Blood gas analysis; COPD: Chronic obstructive pulmonary disease; ECMO: Extracorporeal membrane oxygenation; GOT: Glutamic oxaloacetic transaminase; GPT: Glutamate pyruvate transaminase; Hb: Hemoglobin; Hct: Hematocrit; LDH: Lactate dehydrogenase; n.s.: Not significant; PAS: Periodic acid-Schiff; SD: Standard deviation; vV: Veno-venous

\section{Acknowledgements}

We thank Slavia Schuemann for the excellent technical assistance in this project.

\section{Authors' contributions}

NM contributed to the conceptualization. RN contributed to the data curation. RN and FG contributed to the formal analysis. $\mathrm{NM}$ and $\mathrm{AH}$ contributed to the funding acquisition. $\mathrm{RN}$ and $\mathrm{AK}$ contributed to the investigation. FG, AK, and NM contributed to the methodology. CK, UM, and NM contributed to the project administration. CK and UM contributed to the resources. FG, AH, and NM supervised the study. EB contributed to the validation. RN wrote the original draft. EB and NM wrote, reviewed, and edited the manuscript. All authors read and approved the final manuscript.

\section{Funding}

This research work was supported by German KFO 311 Deutsche Forschungs-gemeinschaft (DFG) Grant.

\section{Availability of data and materials}

The datasets used or analyzed during the current study are available from the corresponding author on reasonable request.

\section{Ethics approval and consent to participate}

This study was performed in compliance with the German Animal Protection Law (TierSchG) and was approved by the local animal welfare committee (Lower Saxony State Office for Consumer Protection and Food Safety, Protocol TSA 33.12-42502-04-16/2250).

\section{Consent for publication}

Not applicable

\section{Competing interests}

The authors declare that they have no competing interests.

\footnotetext{
Author details

${ }^{1}$ Department of Cardiothoracic, Transplantation and Vascular Surgery, Hannover Medical School, Hannover, Germany. ${ }^{2}$ Department of Nephrology, Hannover Medical School, Hannover, Germany. ${ }^{3}$ Department of Pneumology, Hannover Medical School, Hannover, Germany. ${ }^{4}$ Clinic of Cardiothoracic Surgery, University Clinic of Würzburg, Oberdürrbacher Strasse 6, 97080 Würzburg, Germany.
}

Received: 29 August 2019 Accepted: 27 November 2019

Published online: 16 December 2019

References

1. Tarzia V, Bortolussi G, Bianco R et al (2015) Extracorporeal life support in cardiogenic shock: impact of acute versus chronic etiology on outcome. J Thorac Cardiovasc Surg 150:333-340. https://doi.org/10.1016/j.jtcvs.2015.02.043

2. Kozinn J, Wrisinger CW (2019) ECMO for adults with severe respiratory failure. Mo Med 116:58-62

3. Tsiouris A, Budev MM, Yun JJ (2018) Extracorporeal membrane oxygenation as a bridge to lung transplantation in the United States: a multicenter survey. ASAIO J 64:689-693. https://doi.org/10.1097/MAT.0000000000000731 
4. Combes A, Hajage D, Capellier G et al (2018) Extracorporeal membrane oxygenation for severe acute respiratory distress syndrome. N Engl J Med 378:1965-1975. https://doi.org/10.1056/NEJMoa1800385

5. Vaquer S, de Haro C, Peruga P et al (2017) Systematic review and meta-analysis of complications and mortality of venovenous extracorporeal membrane oxygenation for refractory acute respiratory distress syndrome. Ann Intensive Care 7 : 1-13. https://doi.org/10.1186/s13613-017-0275-4

6. Kuhl T, Michels G, Pfister R et al (2015) Comparison of the Avalon dual-lumen cannula with conventional cannulation technique for venovenous extracorporeal membrane oxygenation. Thorac Cardiovasc Surg 63:653-662. https://doi.org/ 10.1055/s-0035-1549359

7. Chimot L, Marqué S, Gros A et al (2013) Avalon@ bicaval dual-lumen cannula for venovenous extracorporeal membrane oxygenation: survey of cannula use in france. ASAIO J 59:157-161. https://doi.org/10.1097/MAT.0b013e31827db6f3

8. Hayes D, McConnell PI, Preston TJ et al (2013) Active rehabilitation with venovenous extracorporeal membrane oxygenation as a bridge to lung transplantation in a pediatric patient. World J Pediatr 9:373-374. https://doi.org/10. 1007/s12519-013-0437-x

9. Colombier S, Prêtre R, lafrate M, Niclauss L (2016) Double-stage venous cannulation combined with Avalon ${ }^{\oplus}$ cannula for potential prolongation of respiratory ECMO in end-stage pulmonary disease. Perfus (United Kingdom) 31:593-597. https://doi.org/10.1177/0267659116641483

10. Gothner M, Buchwald D, Strauch JT et al (2015) The use of double lumen cannula for veno-venous ECMO in trauma patients with ARDS. Scand J Trauma Resusc Emerg Med 23:1-6. https://doi.org/10.1186/s13049-015-0106-2

11. Bermudez CA, Rocha RV, Sappington PL et al (2010) Initial experience with single cannulation for venovenous extracorporeal oxygenation in adults. Ann Thorac Surg 90:991-995. https://doi.org/10.1016/j.athoracsur.2010.06.017

12. Madrahimov N, Khalikov A, Boyle EC et al (2018) Veno-venous extracorporeal membrane oxygenation in a mouse. J Vis Exp:e58146-e58146. https://doi.org/10.3791/58146

13. Natanov R, Gueler F, Falk CS et al (2018) Blood cytokine expression correlates with early multi-organ damage in a mouse model of moderate hypothermia with circulatory arrest using cardiopulmonary bypass. PLoS One 13:1-17. https://doi.org/10.1371/journal.pone.0205437

14. Madrahimov N, Boyle EC, Gueler F et al (2017) Novel mouse model of cardiopulmonary bypass. Eur J Cardiothorac Surg: 1-8. https://doi.org/10.1093/ejcts/ezx237

15. Hayes D, Yates AR, Duffy VL et al (2014) Rapid placement of bicaval dual-lumen catheter in a swine model of venovenous ECMO. J Investig Surg 27:27-31. https://doi.org/10.3109/08941939.2013.826311

16. Hayes D, Preston TJ, Davis IC et al (2013) Contrast transthoracic echocardiography and the placement of a bicaval duallumen catheter in a swine model of venovenous extracorporeal membrane oxygenation. Artif Organs 37:574-580. https://doi.org/10.1111/aor.12044

17. Speggiorin S, Robinson SG, Harvey C et al (2015) Experience with the Avalon bicaval double-lumen veno-venous cannula for neonatal respiratory ECMO. Perfus (United Kingdom) 30:250-254. https://doi.org/10.1177/0267659114540020

18. Jarboe MD, Gadepalli SK, Church JT et al (2018) Avalon catheters in pediatric patients requiring ECMO: placement and migration problems. J Pediatr Surg 53:159-162. https://doi.org/10.1016/j.jpedsurg.2017.10.036

19. Wetz AJ, Richardt EM, Schotola $\mathrm{H}$ et al (2017) Haptoglobin and free haemoglobin during cardiac surgery - is there a link to acute kidney injury? Anaesth Intensive Care 45:58-66

20. Villa G, Katz N, Ronco C (2015) Extracorporeal membrane oxygenation and the kidney. CardioRenal Med 6:50-60. https://doi.org/10.1159/000439444

21. Antonucci E, Lamanna I, Fagnoul D et al (2016) The impact of renal failure and renal replacement therapy on outcome during extracorporeal membrane oxygenation therapy. Artif Organs 40:746-754. https://doi.org/10.1111/aor.12695

22. Darby PJ, Kim N, Hare GMT et al (2013) Anemia increases the risk of renal cortical and medullary hypoxia during cardiopulmonary bypass. Perfusion 28:504-511. https://doi.org/10.1177/0267659113490219

23. Ranucci M, Aloisio T, Carboni G et al (2015) Acute kidney injury and hemodilution during cardiopulmonary bypass: a changing scenario. Ann Thorac Surg 100:95-100. https://doi.org/10.1016/j.athoracsur.2015.02.034

24. Vermeulen Windsant IC, de Wit NCJ, Sertorio JTC et al (2014) Hemolysis during cardiac surgery is associated with increased intravascular nitric oxide consumption and perioperative kidney and intestinal tissue damage. Front Physiol 5: 1-9. https://doi.org/10.3389/fphys.2014.00340

\section{Publisher's Note}

Springer Nature remains neutral with regard to jurisdictional claims in published maps and institutional affiliations.

\section{Submit your manuscript to a SpringerOpen ${ }^{\circ}$ journal and benefit from:}

- Convenient online submission

- Rigorous peer review

- Open access: articles freely available online

- High visibility within the field

- Retaining the copyright to your article

Submit your next manuscript at $>$ springeropen.com 